\title{
Numerical Evaluation of Hygrothermal Behavior and Moisture Risk for Multi-layer Wall in the Hot and Humid Southern China Area
}

\author{
Aimin Fang ${ }^{1,2}$, Youming Chen ${ }^{1,2}$ \\ ${ }^{1}$ College of Civil Engineering, Hunan University, Changsha, Hunan 410082, China \\ ${ }^{2}$ Key Laboratory of Building Safety and Energy Efficiency of the Ministry of Education, Hunan \\ University, Changsha, Hunan 410082, China
}

\begin{abstract}
The water content is discontinuous at the interface between two materials, which is an obstacle when modelling a multi-layer wall. We have developed a coupled heat and moisture (CHM) transfer model in the porous and hygroscopic wall materials, it has continuous driving potentials of capillary pressure and temperature that allow hygrothermal simulation within multi-layer walls. The results calculated with the proposed model implemented on COMSOL are in good agreement with the results of HAMSTAD project. Then, hourly dynamic simulation of the hygrothermal performance for external insulation wall is carried out during cooling season in Guangzhou. The results show that moisture accumulation risk is great at the interface between insulation and mortar. Cooling load transmitted through the wall calculated with CHM model is about $10.7 \%$ larger than that done with transient heat conduction (TH) model. The sum of latent load accounts for $9.7 \%$ of the total cooling load. A thermal gradient, in addition to the heat transfer, can also generate a mass flow. Both water vapor diffusion with phase change and thermo-migration of the liquid phase all contribute to heat transport.
\end{abstract}

\section{Introduction}

Continuous exposure to hot and humid environment may greatly affect the thermal performance of building materials, particularly thermal insulation, and has a negative impact on building energy consumption. The materials used in constructions are mainly characterized by porous and hygroscopic properties that make them sensitive to the water vapor contained in the surrounding air. A "dry" porous medium can provide good insulation due to its low thermal conductivity, but the presence of water vapor may pose a strong degradation of thermal performance and have a negative impact on its durability. In China, energy-saving policies and thermal regulations have led to the introduction of thermal insulation to reduce the heating or cooling transmission load (China Architecture and Building Press, Ministry of Housing and Urban-Rural Development of the People's Republic of China, 2016). However, the installation of insulation in new buildings or renovation of existing buildings can create confined living spaces with very tight walls, fine airtightness and little renewal of indoor air. These changes to the envelope can cause phenomena that related to moisture: mould growth, degradation of air quality, condensation in the walls, which can lead to decrease in the durability of the materials and their thermal performance.

The theoretical bases for modeling the coupled phenomena of heat and mass transport in a porous medium have been developed since 1957 by Philip and de Vries (Philip, J. and De Vries, D., 1957) and later by Luikov (Luikov, A.V., 1975), Whitaker (Whitaker, S., 1977) and Künzel (Künzel, H.M., 1995)0, the Künzel model is used in the WUFI software which represents one of the most used commercial tools in the 1D and 2D modeling of heat and moisture transfer. However, the physical parameters or coefficients in the governing equations proposed by Philip and de Vries, Luikov and Whitaker are difficult to obtain, and the solution is very complicated. The Philip and de Vries's model underestimates the flow of moisture by approximately five orders of magnitude (Bouddour, A., Auriault, J., Mhamdialaoui, M., et al., 1998). The disadvantage of Luikov's model is the presence of phase change factor in energy balance equation which is not derived from a physical law and the empirical determination of this factor is difficult. Furthermore, thermo-migration of the liquid phase is neglected in Künzel model. Künzel simplifies the moisture transfer to a pure diffusive model: vapor transport and surface liquid diffusion are described with Fick's diffusion law, with water vapor partial pressure as driving potential for vapor flow and relative humidity for the surface diffusion (Guizzardi, M., 2014). In recent years, many studies have been launched in many countries to better understand the phenomena associated with hygrothermal transfer and to adapt or correlate models of hygrothermal coupling calculations. Odgaard et al. (2018) evaluated the influence of hygrothermal phenomena on their characteristics and durability. Tariku et al. (2010) conducted a study aimed at characterizing and modeling the thermo-hydric behavior of porous materials, showing their particular behavior having an influence on the indoor hygrothermal comfort and the energy performance of the building.

However, in spite of these numerous developments, several scientific obstacles remain to be raised to better apprehend the phenomena. On the one hand, discrepancies between physical measurements and numerical simulations have been observed in many projects. On the other hand, there are trade-offs and uncertainties, as insulation renovation can be detrimental 
in other areas, such as moisture accumulation and degradation of thermal performance depending on the location of the building and the climate. This work is devoted to the study of hygrothermal performance and moisture risk for multi-layer walls renovated with external insulation. Our work aims at a better understanding of the coupled heat and moisture transfer within porous and hygroscopic building materials.

\section{Climatic characterization of the study area}

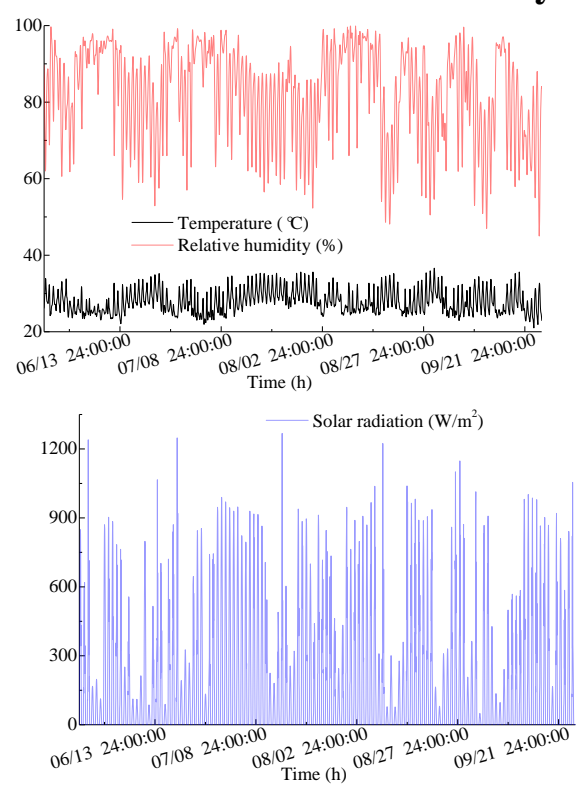

Figure 1: Relative humidity, temperature and solar radiation for the studied cooling season.

Guangzhou, the largest city located in hot summer and warm winter region of China is chosen for analysis. Located at $23^{\circ} 17$ North longitude and $113^{\circ} 33$ East longitude, it is bordered to the north by the South China Sea. Figure 1 shows the values of the ambient temperature, ambient relative humidity and the total solar radiation on the horizontal plane, respectively. The climate of Guangzhou is quite hot and humid in summer, warm in winter, characterized by an average annual temperature of $22.2^{\circ} \mathrm{C}$ with $28.8^{\circ} \mathrm{C}$ in July and $13.9^{\circ} \mathrm{C}$ in January. Moisture is high in both winter and summer, the monthly average relative humidity exceeds $76 \%$ with a maximum average of $84.5 \%$ in June and a minimum average of $60.8 \%$ in October. The highest monthly mean value of the total solar radiation on the horizontal plane reaches $431.5 \mathrm{~W} / \mathrm{m}^{2}$ in July, and the average monthly solar radiation intensity is $340.6 \mathrm{~W} / \mathrm{m}^{2}$ (China Meteorological Bureau, Climate Information Center, Climate Data Office and Tsinghua University, Department of Building Science and Technology , 2005).

\section{Methodology for hygrothermal performance}

\section{Model assumptions}

The solid phase is composed of dry material, considered as homogeneous and non-deformable. The chemical reactions in the material are neglected. The effects of gravity are neglected. Materials is never more than capillary moisture saturated. No hysteresis. An immediate thermodynamic equilibrium is established between the liquid phase and the gas phase. The gas phase is composed of water vapor and air, both considered as ideal gases. In porous capillary media, convective heat transfer is neglected due to low value of Reynolds number. The liquid phase is composed of water, considered as incompressible. The temperature of the material remains above the freezing temperature of the water. At the interfaces between two materials, the contact is perfect, no contact resistance is taken into consideration.

\section{Mathematical models: energy and mass balance}

In building materials, moisture can exist in the pores as two thermodynamic states: liquid or water vapor. Depending on the driving force that governs the model of transfer in a porous material, moisture can pass in different forms and in different ways.

Diffusion of water vapor:

$$
\frac{\partial \omega_{v}}{\partial t}+\nabla g_{v}=-G_{l, v}
$$

where, $\omega_{v}$ is the moisture content in vapor phase, $\mathrm{kg} / \mathrm{m}^{3}$; $t$ represents time, $\mathrm{s} ; g_{v}$ is the moisture flow rate in vapor phase, $\mathrm{kg} /\left(\mathrm{m}^{2} \cdot \mathrm{s}\right) ; G_{l, v}$ is the source term for the amount moisture exchange between gaseous water and liquid water, $\mathrm{kg} /\left(\mathrm{m}^{3} \cdot \mathrm{s}\right)$.

Diffusion of liquid water:

$$
\frac{\partial \omega_{l}}{\partial t}+\nabla g_{l}=G_{l, v}
$$

where, $\omega_{l}$ is the moisture content in liquid phase, $\mathrm{kg} / \mathrm{m}^{3}$; $g_{l}$ is the moisture flow rate in liquid phase, $\mathrm{kg} /\left(\mathrm{m}^{2} \cdot \mathrm{s}\right)$. The above two equations are combined to obtain the total moisture transfer equilibrium equation of the gas-liquid interface in the porous medium material:

$$
\frac{\partial \omega_{v}}{\partial t}+\frac{\partial \omega_{l}}{\partial t}+\nabla g_{v}+\nabla g_{l}=0
$$

The above Eq.(3) can be simplified as:

$$
\frac{\partial \omega}{\partial t}+\nabla g_{v}+\nabla g_{l}=0
$$

where, moisture content $\omega$ is the total amount of moisture per unit volume of porous material, in $\mathrm{kg} / \mathrm{m}^{3}$;

$$
\omega=\omega_{v}+\omega_{l}
$$

The moisture content of the wall material is characterized by the water retention curve, the moisture content can be expressed as a function of capillary pressure.

$$
\omega=f\left(p_{c}\right)
$$

where, $p_{c}$ is capillary pressure, $\mathrm{Pa}$; The moisture balance equation for a porous media can be given as:

$$
\frac{\partial \omega}{\partial t}=\frac{\partial \omega}{\partial p_{c}} \frac{\partial p_{c}}{\partial t}=\zeta \frac{\partial p_{c}}{\partial t}
$$

where, $\zeta$ is defined as the slope of the water retention curve. Within the porous material, without air flow, the moisture can be transported in the form of vapor by 
diffusion and in liquid form by capillarity. The diffusion of water vapor is generated by a vapor partial pressure gradient. The diffusive vapor flow is described by Fick's law.

$$
\mathrm{g}_{v}=-\delta_{v} \nabla p_{v}
$$

At the pore scale, the equilibrium between the liquid and vapor phases is described by the Kelvin law, which restricts the suction pressure to temperature and relative humidity. The capillary pressure is related to the relative humidity via the Kelvin law given by the expression:

$$
p_{c}=-\rho_{l} R_{D} T \ln (\varphi)
$$

where, $\rho_{l}$ is the density of liquid water, $\mathrm{kg} / \mathrm{m}^{3} . R_{D}$ represents the water vapor gas constant, $\mathrm{J} /(\mathrm{kg} \cdot \mathrm{K}) ; \varphi$ is the relative humidity. The derivative of relative humidity with respect to capillary pressure gives:

$$
\frac{\partial \varphi}{\partial p_{c}}=\frac{1}{\rho_{l} R_{D} T} \exp \left(-\frac{p_{c}}{\rho_{l} R_{D} T}\right)
$$

Substituting Eq. (8) into Eq. (9) yields:

$$
\frac{\partial \varphi}{\partial p_{c}}=\frac{1}{\rho_{l} R_{D} T} \exp \left(-\frac{\rho_{l} R_{D} T \ln (\varphi)}{\rho_{l} R_{D} T}\right)=\frac{-\varphi}{\rho_{l} R_{D} T}
$$

Liquid water in a porous medium can migrate under the effect of a capillary pressure gradient. The flow of liquid water can be expressed by Darcy's law.

$$
g_{l}=K_{l} \nabla p_{c}
$$

In fact, to guarantee the applicability of Eq. (4), only one pressure variable is used as a driving potential for moisture transfer, thus the equivalence between $\nabla \mathrm{p}_{v}$ and $\nabla \mathrm{p}_{c}$ need to be established.

$$
\begin{aligned}
& \nabla p_{v}=\frac{\partial p_{v}}{\partial x} \\
& =\frac{\partial\left(\varphi p_{\text {sat }}\right)}{\partial x} \\
& =\left(\varphi \frac{\partial p_{\text {sat }}}{\partial x}+p_{\text {sat }} \frac{\partial \varphi}{\partial x}\right) \\
& =\left(\varphi \frac{\partial p_{\text {sat }}}{\partial T} \frac{\partial T}{\partial x}+p_{\text {sat }} \frac{\partial \varphi}{\partial p_{\mathrm{c}}} \frac{\partial p_{\mathrm{c}}}{\partial x}\right) \\
& =\left(\varphi \frac{\partial p_{\text {sat }}}{\partial T} \nabla T-p_{\text {sat }} \frac{\varphi}{\rho_{l} R_{D} T} \nabla p_{\mathrm{c}}\right)
\end{aligned}
$$

The saturation vapor pressure can be calculated as a function of the temperature according to the following empirical expression ( Lin, M.W. et al., 2006):

$$
p_{\text {sat }}=610.5 \exp \left(\frac{17.269 T}{237.3+T}\right)
$$

Generally, the thermohydric properties differ greatly from one material to the other. The choice of the variable capillary pressure guarantees the continuity of this potential at the wall inter-layers.

Substituting Eq. (12) and Eq. (13) into Eq. (4), this leads to the following transfer equation for moisture:

$$
\zeta \frac{\partial p_{c}}{\partial t}=\nabla\left\lfloor\delta_{v} \varphi \frac{\partial p_{s a t}}{\partial T} \nabla T+\left(-K_{l}-\delta_{v} p_{s a t} \frac{\varphi}{\rho_{l} R_{D} T}\right) \nabla p_{c}\right\rfloor
$$

\section{Energy transfer equilibrium equation}

The energy and mass balances are based on the same principle: The sum of the temporal variation of the quantity of energy or of mass and the divergences of energy (mass) flow is equal to zero. We assume that there is no change in the total pressure and therefore air transfers in the porous materials are not taken into account. The internal energy of the control volume is composed of the internal energy of the solid matrix of the material and the internal energy of the liquid phase contained in the pores: The derivative of the internal energy with respect to time gives:

$$
\frac{\partial}{\partial t}\left(\rho_{m} c_{p, m} T+h_{v} \omega_{v}+h_{l} \omega_{l}\right)=-\nabla\left(Q+h_{v} g_{v}+h_{l} g_{l}\right)
$$

where, $\rho_{m}$ is the dry density of the material, $\mathrm{kg} / \mathrm{m}^{3} ; c_{p, m}$ refers to the specific heat capacity of material in dry state, $\mathrm{J} /(\mathrm{kg} \cdot \mathrm{K}) ; \quad h_{l}$ represents the specific enthalpy of liquid water, $\mathrm{J} / \mathrm{kg} ; h_{v}$ represents the specific enthalpy of water vapor, $\mathrm{J} / \mathrm{kg} ; Q$ is heat flux density transmitted through heat conduction, $\mathrm{W} / \mathrm{m}^{2}$.

The flow of heat conduction follows the law of Fourier:

$$
Q=-\lambda \nabla T
$$

where, $\lambda$ is the thermal conductivity of the material, $\mathrm{W} /(\mathrm{m} \cdot \mathrm{K})$. The flow of vapor entering the representative volume is not necessarily equal to the outgoing flow, but the difference of the two mass flows corresponds to the quantity of water stored in adsorbed form. In practical applications, since the density of liquid water $\left(\rho_{l}=1000 \mathrm{~kg} / \mathrm{m}^{3}\right)$ is about 1666 times greater than that of water vapor $\left(\rho_{v}=0.6 \mathrm{~kg} / \mathrm{m}^{3}\right)$, the variation in total amount of moisture can be considered as liquid water. Taking the expression of the moisture flow given by Eqs. (8) 、 (12) and (13), the heat conservation equation is finally written as:

$$
\begin{aligned}
& \left(\rho_{m} c_{p, m}+\omega c_{p, l}\right) \frac{\partial T}{\partial t}=\nabla \mid\left(\lambda+h_{v} \delta_{v} \varphi \frac{\partial p_{\text {sat }}}{\partial T}\right) \nabla T+ \\
& \left.\left(-h_{l} K_{l}-h_{v} \delta_{v} p_{s a t} \frac{\varphi}{\rho_{l} R_{D} T}\right) \nabla p_{c}\right]
\end{aligned}
$$

The modeling of the heat and mass transfers of the case study was carried out in the COMSOL software. COMSOL is a simulation environment based on the finite element method that allows to study a wide range of physical phenomena. The mathematical interface PDE module makes it possible to adapt the model described previously in the form of coefficients and to ensure the coupling between the studied heat and mass transfer.

\section{Transient heat flow through composite wall without considering moisture transfer}

At present, the most commonly used transient heat conduction (TH) model is the unsteady state heat conduction equation, the one-dimensional equation governing transient heat flow through a composite wall is expressed as (Ozel, M., 2014) : 


$$
\rho_{m} c_{p, m} \frac{\partial T}{\partial t}=\lambda \nabla^{2} T
$$

\section{Conditions at the interfaces in the building}

This study focuses on the numerical modeling of the hygrothermal behavior of a multi-layer wall in one dimension. As shown in Figure 2, from the interior to the exterior, the composite wall consists of $20 \mathrm{~mm}$ mortar, $240 \mathrm{~mm}$ brick, $20 \mathrm{~mm}$ mortar and $80 \mathrm{~mm}$ external insulation material (node 5 is directly subjected to the outdoor environment, node 1 is in contact with the indoor environment)

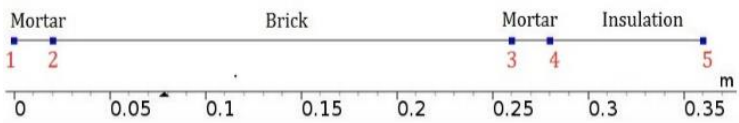

Figure 2: Schematic diagram of the studied multilayer wall.

Convective heat and mass exchanges are considered at the level of wall faces in contact with the indoor and outdoor environments. The heat flux density on the outdoor side surface is equal to the heat transmitted to the atmosphere by convection accompanied by latent heat transfer. In the same way, moisture transported to the wall surface is conducted through convective mass exchange with the atmosphere. The outdoor side boundary conditions for heat $\left(q_{h, e}\right)$ and mass $\left(g_{m, e}\right)$ transfers are then written as follows:

$$
\begin{gathered}
q_{h, e}=h_{e}\left(T_{e}-T_{\text {surfe }}\right)+h_{v} \cdot g_{m, e}+\alpha \cdot q_{\text {solar }} \\
g_{\mathrm{m}, e}=\beta_{p, e}\left(\varphi_{e} p_{\text {sat }, e}-\varphi_{\text {surfe }} p_{\text {sat }, \text { surfe }}\right)
\end{gathered}
$$

where, $\beta_{p, e}$ is convective mass exchange coefficient, $\mathrm{kg} /\left(\mathrm{m}^{2} \cdot \mathrm{s} \cdot \mathrm{Pa}\right) ; h_{e}$ is convective heat exchange coefficient, $\mathrm{W} /\left(\mathrm{m}^{2} \cdot \mathrm{K}\right) ; \alpha$ is the solar radiation absorption rate; $q_{\text {solar }}$ is heat flux density of solar radiation received on the outside surface of the wall, $\mathrm{W} / \mathrm{m}^{2}$. At the level of transfers between the surface of the inner facing and the indoor air, the wall exchanges heat and water vapor by convection. The indoor air set-point temperature and relative humidity are assumed equal to $26^{\circ} \mathrm{C}$ and $60 \%$, respectively. The boundary conditions of heat and moisture transfer for the inside of the wall are similar to that of the outdoor side, but there is no solar radiation to the indoor side. The boundary conditions on the indoor side are not listed here.

\section{Numerical validation}

The model of heat and mass transfer in the multilayer porous wall developed under COMSOL is then validated by results from the HAMSTAD benchmark (Hagentoft, C., 2002). The benchmark 5 is related to the hygrothermal behavior of a multilayer wall composed of materials with different properties, the hygrothermal properties differ greatly from one material to the other. The duration of the simulation is 60 days. The initial temperature is $25^{\circ} \mathrm{C}$ and the initial relative humidity is $60 \%$ throughout the whole construction. At $t>0$, the internal thermal boundary condition is fixed at $20^{\circ} \mathrm{C}$, the temperature outside at $0{ }^{\circ} \mathrm{C}$. Also, $60 \%$ and $80 \%$ relative humidity are applied to the two surfaces of the wall respectively and the evolution of the moisture content over time is monitored. Heat and mass transfer coefficients at interior and exterior sides have the values of $8 \mathrm{~W} /\left(\mathrm{m}^{2} \mathrm{~K}\right)$ and $25 \mathrm{~W} /\left(\mathrm{m}^{2} \mathrm{~K}\right) ; 5.8823 \times 10^{-8} \mathrm{~s} / \mathrm{m}$ and

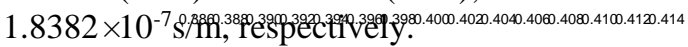
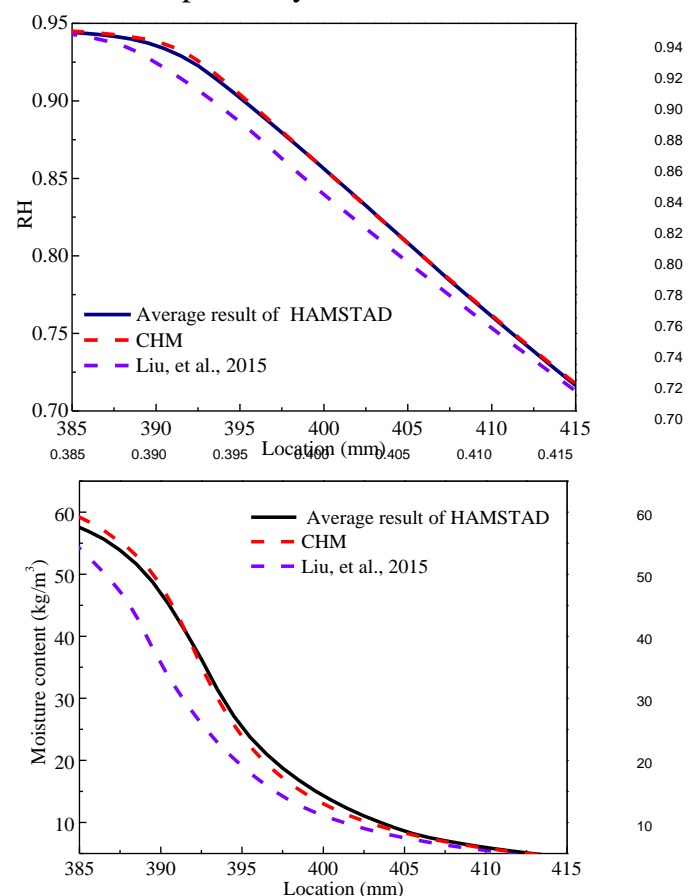

Figure 3: Relative humidity $(\mathrm{RH})$ and moisture content profile through the wall on day 60.

Figure 3 illustrates the results of the relative humidity and the moisture content of the wall calculated with the adopted model, also the results in Figure 3 are presented on part of the thickness of the wall after 60 days. The results of the proposed model are first compared to the results obtained by (Liu, et al., 2015), they took relative humidity and temperature as driving potentials to establish the coupled heat and moisture transfer model, then compared to the average results of the HAMSTAD project. The results calculated with the proposed model in this study are more close to the average results of the HAMSTAD project than those of (Liu, et al., 2015), thus validating our model.

\section{Results and discussion}

\section{Evolution of relative humidity}

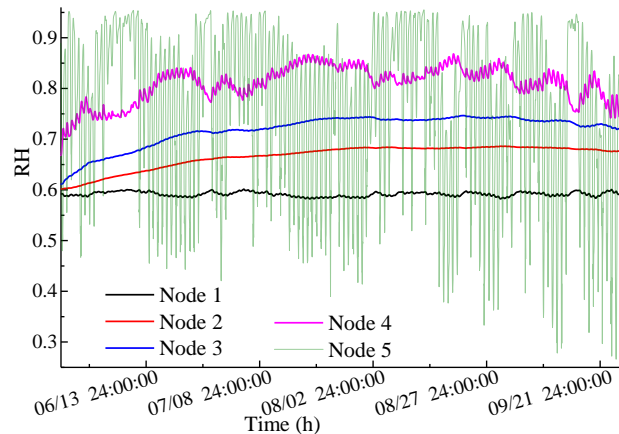

Figure 4: Relative humidity $(R H)$ of the inter-layer nodes. 
Figure 4 shows a comparison of the relative humidity of the multi-layer wall obtained during the whole cooling period. The shape of the humidity curve for exterior insulation wall does not follow the same evolution, interlayer nodes have diverse surface relative humidity present. The relative humidity at the wall outside surface fluctuations frequent in a day, this clear variation could be the product of the large moisture exchanges with the outside environment since outside surface is directly exposed to hot and humid outdoor environment. The exterior insulation wall outside surface (node 5 in Figure 2) has a larger relative humidity than the others. The humidity at node 4 continues to increase with monthly average relative humidity exceeds $80 \%$ and a maximum relative humidity reaches $86.8 \%$, which means that there is a large risk of moisture accumulation. Indeed most materials used in the building are porous, containing water in vapor or liquid form. As the porous and hygroscopic wall materials could have the capacity to absorb more or less moisture depending on hygroscopic properties, thus damping the variations of relative humidity at the wall inter-layer. It is found that relative humidity shows a remarkable downward trend between node 3 and node 2 in the direction from the outside to the inside. Relative humidity hardly changes for the inner surface (node 1) of the wall, it is almost constant around $60 \%$.

\section{Cooling load transmitted through the wall}

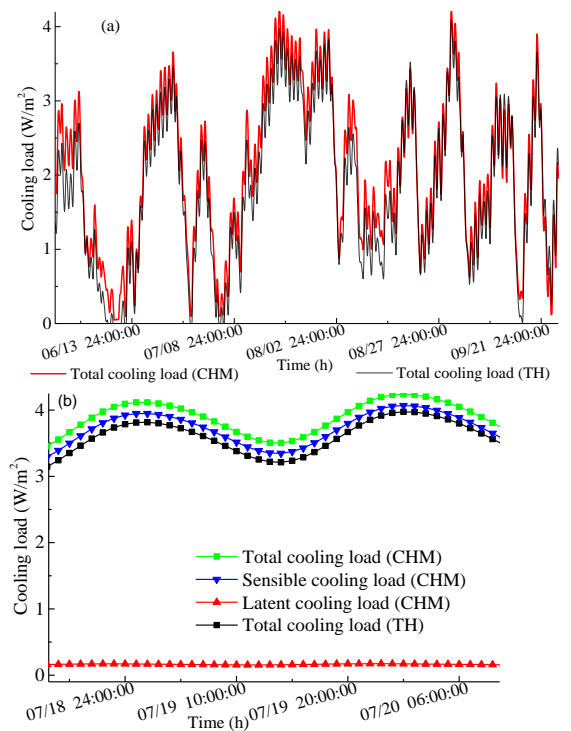

Figure 5: Cooling loads transmitted through the wall use CHM and TH models.

Figure 5 demonstrates cooling load transmitted through the south-facing wall using the two studied CHM and TH heat transfer methods throughout the cooling season. Cooling load transmitted through the wall calculated with CHM model is about $10.7 \%$ larger than that of transient heat conduction (TH) model in Figure 5(a). The difference between the two studied total cooling load curves is obvious, which proves that water vapor exchange acting on the porous media is able to affect the wall transmitted heat flux. In order to be able to compare our results, we choose to study the response of the wall after a series of two relatively identical summer days, with a stable external mean temperature and similar humidity variation. This is the cooling period from $7 / 18$ to $7 / 20$ for the insulation renovation study in Figure 5(b). The impact of taking into account or not the heat of water vapor phase change is studied by two calculations: CHM model with latent heat, the TH model without latent heat. The sum of latent load accounts for $9.7 \%$ of the total cooling load. The phase change latent heat caused by absorption and desorption moisture of porous media which contributes to total heat transfer quantity can't be neglected. In porous and hygroscopic building materials, heat and moisture are two transport phenomenon that may significantly degrade the insulation properties of a multilayer wall. It is worth noting that the mechanisms of moisture transport in the material are not taken into account in the TH model. This can lead to an under-evaluation of air-conditioning demand in the building's energy simulation.

For moist porous media, the dominant heat transfer is that by conduction which is often coupled to a state of phase change due to the amount of continuous water in the medium. Thus the transfer of heat flow is reflected in a variation of enthalpy of the material under consideration. This variation is governed mainly by the Fourier law and the gradient of the heat flux carried by the moisture flux. In practice, these transport processes usually operate in a coupled fashion. A thermal gradient, in addition to the heat transfer, can also generate a mass flow. Furthermore, mass transfer in porous media result in energy transfer. On one hand, water vapor diffusion with phase change contributes to heat transport. On the other hand, the thermo-migration of the liquid phase is also involved in the overall heat transfer as the third term described in Eq. (18).

\section{Conclusions}

A model of coupled heat and mass transfer equations is established and validated. The model is then applied to the case of external insulation wall in order to analyze and quantify more precisely the influence of moisture transfer on the thermal behavior of the wall. Moisture accumulation risk is great at the interface between insulation and mortar. Cooling load transmitted through the wall calculated with CHM model is about $10.7 \%$ larger than that of transient heat conduction $(\mathrm{TH})$ model. The sum of latent load accounts for $9.7 \%$ of the total cooling load. A thermal gradient, in addition to the heat transfer, can also generate a mass flow. Both water vapor diffusion with phase change and thermo-migration of the liquid phase all contribute to heat transport. The impact of mass transfer on heat transfer is highlighted for the porous and hygroscopic wall material.

\section{Acknowledgement}

This research has been supported by the China National Key R\&D Program during the 13th Five-year Plan Period (Grant No. 2017YFC0702201). 


\section{References}

Bouddour, A., Auriault, J., Mhamdialaoui, M., et al. (1998). Heat and mass transfer in wet porous media in presence of evaporation--condensation. International Journal of Heat \& Mass Transfer 41(15):2263-2277.

China Architecture and Building Press, Ministry of Housing and Urban-Rural Development of the People's Republic of China (2016) (in Chinese). Code for thermal design of civil building (GB50176-2016).

China Meteorological Bureau, Climate Information Center, Climate Data Office and Tsinghua University, Department of Building Science and Technology (2005) (in Chinese). China Standard Weather Data for Analyzing Building Thermal Conditions. China Architecture and Building Press, Beijing .

Guizzardi, M. (2014). Hygrothermal performance assessment of novel interior insulation solutions. ETH-Zürich, Switzerland

Hagentoft, C. (2002). HAMSTAD-Final report: methodology of HAM-modeling, Report R-02: 8 . Gothenburg, Department of Building Physics, Chalmers university of Technology .

Künzel, H.M. (1995). Simultaneous heat and moisture transport in building components. Fraunhofer Institute of Building Physics.

Lin, M.W., Berman J. B. , Khoshbakht M. , et al. (2006). Modeling of moisture migration in an FRP reinforced masonry structure. Building \& Environment 41 (5) :646-656.

Luikov, A.V. (1975). Systems of differential equations of heat and mass transfer in capillary-porous bodies (review). International Journal of Heat and Mass Transfer 18(1): 1-14.

Liu, X.W., Chen, Y.M., Ge, H., et al. (2015). Determination of optimum insulation thickness for building walls with moisture transfer in hot summer and cold winter zone of China. Energy \& Buildings 109(12): 361-368.

Ozel, M. (2014). Effect of insulation location on dynamic heat-transfer characteristics of building external walls and optimization of insulation thickness. Energy \& Buildings 72(72):288-295.

Odgaard, T., Søren, P. and Rode, C. (2018). Interior insulation - Experimental investigation of hygrothermal conditions and damage evaluation of solid masonry façades in a listed building. Building \& Environment 129:1-14.

Philip, J. and De Vries, D. (1957). Moisture movements in porous materials under temperature gradients. Eos Transactions American Geophysical Union 38(2):222-232.

Tariku, F., Kumaran, K. and Fazio, P. (2010). Integrated analysis of whole building heat, air and moisture transfer. International Journal of Heat \& Mass Transfer 53(15):3111-3120.

Whitaker, S. (1977). Simultaneous heat, mass and momentum transfer in porous media: a theory of drying. Advanced in Heat Transfer 13(8):119-123. 